\title{
Genome Compositional Organization in Gars Shows More Similarities to Mammals than to Other Ray-Finned Fish
}

RADKA SYMONOVÁ1,2,3* ZUZANA MAJTÁNOVÁ ${ }^{1,2}$, LENIN ARIAS-RODRIGUEZ ${ }^{4}$, LIBOR MOŘKOVSKÝ ${ }^{2}$, TEREZA KOŘÍNKOVÁ ${ }^{1}$, LIONEL CAVIN $^{5}$, MARTINA JOHNSON POKORNÁ ${ }^{1,6}$, MARIE DOLEŽÁLKOVÁ ${ }^{1,2}$, MARTIN FLAJŠHANS ${ }^{7}$, ERIC NORMANDEAU ${ }^{8}$, PETR RÁB ${ }^{1}$, AXEL MEYER ${ }^{9}$, AND LOUIS BERNATCHEZ ${ }^{8}$

${ }^{1}$ Laboratory of Fish Genetics, Institute of Animal Physiology and Genetics, The Czech Academy of Sciences, Liběchov, Czech Republic

${ }^{2}$ Department of Zoology, Faculty of Science, Charles University, Prague 2, Czech Republic

${ }^{3}$ Research Institute for Limnology, University of Innsbruck, Mondsee, Austria

${ }^{4}$ División Académica de Ciencias Biológicas, Universidad Juárez Autónoma de Tabasco (UJAT),

Villahermosa, Tabasco, México

${ }^{5}$ Muséum d'Histoire Naturelle, Geneva 6, Switzerland

${ }^{6}$ Department of Ecology, Faculty of Science, Charles University, Prague 2, Czech Republic

${ }^{7}$ Faculty of Fisheries and Protection of Waters, South Bohemian Research Centre of Aquaculture and Biodiversity of HydrocenosesUniversity of South Bohemia in České Budějovice, Vodňany, Czech Republic

${ }^{8}$ IBIS, Department of Biology, University Laval, Pavillon Charles-Eugène-Marchand, Avenue de la Médecine Quebec City, Canada

${ }^{9}$ Chair in Zoology and Evolutionary Biology, Department of Biology, University of Konstanz,

Konstanz, Germany

Conflicts of interest: None

Grant Sponsor: This study was supported by projects P506/11/P596 and 14-02940S of the Czech Science Foundation and by the project between the Academy of Sciences of the Czech Republic and the National Council for Science and Technology of Mexico 167686/204264, the project 43-251468 of the Charles University Grant Agency and Research Support Grant SW 260087/2014, supported by the Ministry of Education, Youth and Sports of the Czech Republic projects "CENAKVA" No. CZ.1.05/2.1.00/01.0024, "CENAKVA II" No. L01205 under the NPU I program. LC was supported partly by the Swiss National Research Fund (200021-140827).

*Correspondence to: Radka Symonová; Research Institute for Limnology, University of Innsbruck, Mondseestraße 9, A-5310 Mondsee, Austria. E-mail: Radka.Symonova@uibk.ac.at 
Genomic GC content can vary locally, and GC-rich regions are usually associated with increased DNA thermostability in thermophilic prokaryotes and warm-blooded eukaryotes. Among vertebrates, fish and amphibians appeared to possess a distinctly less heterogeneous AT/GC organization in their genomes, whereas cytogenetically detectable GC heterogeneity has so far only been documented in mammals and birds. The subject of our study is the gar, an ancient "living fossil" of a basal ray-finned fish lineage, known from the Cretaceous period. We carried out cytogenomic analysis in two gar genera (Atractosteus and Lepisosteus) uncovering a GC chromosomal pattern uncharacteristic for fish. Bioinformatic analysis of the spotted gar (Lepisosteus oculatus) confirmed a GC compartmentalization on GC profiles of linkage groups. This indicates a rather mammalian mode of compositional organization on gar chromosomes. Gars are thus the only analyzed extant ray-finned fishes with a GC compartmentalized genome. Since gars are cold-blooded anamniotes, our results contradict the generally accepted hypothesis that the phylogenomic onset of GC compartmentalization occurred near the origin of amniotes. Ecophysiological findings of other authors indicate a metabolic similarity of gars with mammals. We hypothesize that gars might have undergone convergent evolution with the tetrapod lineages leading to mammals on both metabolic and genomic levels. Their metabolic adaptations might have left footprints in their compositional genome evolution, as proposed by the metabolic rate hypothesis. The genome organization described here in gars sheds new light on the compositional genome evolution in vertebrates generally and contributes to better understanding of the complexities of the mechanisms involved in this process.

\section{INTRODUCTION}

Genomic compositional architecture is a complex and nonrandom organization of DNA bases (AT/GC). Genomes of higher vertebrates can be described as mosaics of compositionally homogeneous DNA domains (sometimes called isochores) with a distinct GC content (Bernardi, 2005). So called "light" domains with lower GC proportions represent the late-replicating genepoor genome "desert" and "heavy" domains with higher GC proportions represent the early-replicating gene-rich genome "core" (Bernardi, 2005). Ray-finned fish genomic architecture with a narrower range in GC proportions differs considerably from mammalian architecture where a broad range of GC proportions occurs (Costantini et al., 2007). Genomes of hundreds of vertebrates, including about 300 fish species, were investigated for genomic base composition (Bernardi and Bernardi, '90). Since gars were never covered in this work, we have analyzed the genome and chromosomes of the spotted gar (Lepisosteus oculatus) and the chromosomes of the tropical gar (Atractosteus tropicus).

Extant gars (Lepisosteiformes) are represented by only seven species divided into two genera- Atractosteus and Lepisosteus (Nelson, 2006; Fig. 1) and are the only survivors of an early radiation of ray-finned fishes highly diversified and widely distributed in the Mesozoic (Cavin, 2010; López-Arbarello, 2012). Gars are believed to be sister to another ancient lineage, Amiiformes, represented by a single extant species, the bowfin (Amia calva) (Grande, 2010). Together with other "ancient fishes" sensu Inoue et al. (2003), gars are sometimes referred to as "living fossils" since they do not appear to have undergone many apparent morphological changes during the last 100 million years (Wright et al., 2012; Sallan, personal communication). Gars and bowfin did not go through any further whole genome duplications (WGD) after the two basal rounds of vertebrate genome duplications (VGD1+2/R1+2) that followed the origin of vertebrates (Fig. 1; Meyer and Malaga-Trillo '99; Vandepoele et al., 2004; Dehal and Boore, 2005). They thus exemplify one of the basal "nonteleost" actinopterygian lineages that branched off the stem lineage before the teleost-specific WGD (TGD; Amores et al., '98; Taylor et al., 2003; Crow et al., 2006) and can therefore serve as an outgroup for exploring evolutionary mechanisms associated with TGD (Amores et al., 2011; Braasch et al., 2016). Ecophysiologically, gars embody a derived group highly specialized to extreme conditions (low oxygen and high-temperature environments) with a metabolic organization that is unique among 


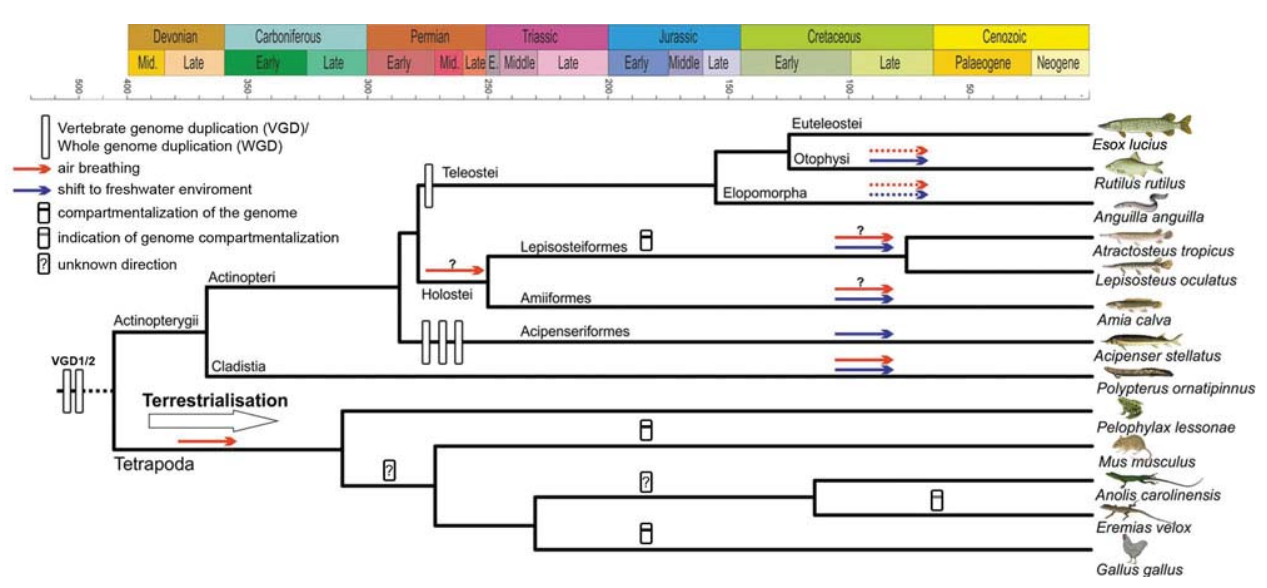

Figure 1. Time-scaled consensus tree of osteichthyes focused on the nonteleost actinopterygians and tetrapod clades in sarcopterygians. Relevant genomic and ecophysiological adaptations are plotted on the tree: vertebrate genome duplications (VGD1/2), following WGD, genome base compartmentalization, air breathing, and terrestrialization. The genomic base compartmentalization is shown to be isolated in gars within the group Actinopterygii. In this phylogeny, we favored a double appearance of heterogeneity within the amniotes (i.e., warm-blooded mammals and birds), although we cannot exclude the possibility that heterogeneity occurred at the amniotes node, then disappeared in several "reptilian" lineages. [Color figure can be viewed at wileyonlinelibrary.com]

fishes in their heightened capacity to synthesize and oxidize glucose, heightened capacity for anaerobic glycolysis, and the low number of oxidative enzymes in gar tissues (Frick et al., 2007). The metabolic organization of their air-breathing organ resembles that of lungs more than that of the swim bladder of most teleosts (Frick et al., 2007). The spotted gar gene expression pattern exhibits many similarities with those of tetrapod genomes (Braasch et al., 2014) and has been utilized to link teleost models influenced by the TGD with the human genome in disease research (Amores et al., 2011; Braasch et al., 2014, 2016).

There has been only limited investigation into gar chromosomes so far, with predominantly just basic karyotypic characteristics and GC-rich ribosomal DNA (rDNA) sites being identified (Ráb et al., '99), along with the very recent research of Braasch et al. (2016) which uncovered the striking conservation between chromosomes of the spotted gar and the chicken. Braasch et al. (2016) found that the karyotypes of these two species differed by merely 17 large fissions, fusions, or translocations.

Independently, rDNA sites in Eukaryotes are generally known as regions of substantial GC enrichment in association with GCbiased gene conversion (Escobar et al., 2011). The genome GC content was initially investigated using analytical centrifugation (Thiery et al., '76). From these studies, later supplemented with genomics, a generally accepted concept arose about the genomic GC compositional heterogeneity in birds and mammals and a substantially less heterogeneous GC organization in fishes and amphibians, with transitional states in reptiles (reviewed in
Bernardi, 2005). A "thermodynamic stability hypothesis" proposed to explain this phenomenon stated that the GC compositional heterogeneity was an adaptation to homeothermy since higher GC levels stabilize coding DNAs along with their RNAs and proteins (Costantini et al., 2009).

We have combined cytogenetics and bioinformatics to obtain cytogenomic insights into the organization of the gar genome and analyzed it further by comparing gars with other vertebrates. In both gar genera, we found GC genome heterogeneity that is uncharacteristic for cold-blooded vertebrates. This was detected bioinformatically and cytogenetically in L. oculatus and cytogenetically in A. tropicus. This led us to reassess the applicability of the conventional cytogenetic method of G-banding in fishes, a technique routinely working so far only in mammals and birds. G-banding visualizes the alternation of gene-rich, early-replicating and gene-poor, late-replicating chromosome regions sensu Bernardi (2005). A comparable banding pattern can be obtained with AT/GC-specific fluorochromes and replication banding, both of which are less prone to interference by artifacts (Sumner, '90). The unavailability of G-banding in lower vertebrates represents a serious obstacle to the study of their cytogenetics, as it makes it impossible to properly identify homologous chromosomes, which is one of the basic prerequisites for all downstream cytogenetic analyses. There are reports showing "G-banding" in fishes but these concluded that the banding pattern is irreproducible and incomparable with patterns routinely produced in mammals (e.g., Medrano et al., '88; Ueda \&t Naoi, 1999; Romanenko et al., 2015; this study). Genuine G-banding 
has been reported to work in eels (Coluccia et al., 2010), cyprinids (Luo, '98), and in tonguefish (Zhuang et al., 2006). However, these results have never been confirmed with any AT/GC-specific fluorochrome staining. Since G-banding provides a reproducible pattern in both gar genera, and genomic data of several fish species are available, we could resolve this issue. We analyzed GC profiles in following teleosts; zebrafish, representing a GC homogenized teleost genome (Costantini et al., 2007), stickleback and pufferfish, both representing more GC heterogeneous genomes as reported by Costantini et al., 2007. Analysis of GC profiles among linkage groups elucidated the relationship between the GC distribution at the sequence and the chromosomal level.

A link between environment, metabolic rate, and genomic GC content has recently been established (e.g., Chaurasia et al., 2011, 2014; Tarallo et al., 2016). Gars, with their physiological adaptations to extreme temperatures and low oxygen conditions (Frick et al., 2007) combined with their peculiar genome organization reported here, represent an excellent model system to bring new insights into this long-lasting and multilevel effort. Namely, the major evolutionary forces driving the GC content variation within and among genomes and the compositional differences between cold- and warm-blooded vertebrates still remained unclear. The GC heterogeneity across the genome results from complex interactions and can be investigated through various techniques involving cytogenetics, ecophysiology, molecular evolution, (phylo)genomics, bioinformatics, regulation of gene expression, DNA methylation, and spatial distribution of DNA in interphase nuclei (Varriale 2014; Mugal et al., 2015a).

\section{MATERIALS AND METHODS}

Blood Culturing and Chromosome Preparation

Details on specimen acquisition are listed in the Supporting Information.

Leucocytes from blood samples of A. tropicus, L. oculatus, A. calva, Polypterus ornatipinnis, and Polypterus lapradei were cultivated, and chromosome spreads were prepared according to the protocol of Fujiwara et al. (2001) with some modifications. Briefly, 0.2-0.5 mL of blood was collected from an anesthetized fish by puncturing the vena cava caudalis using a heparinized syringe. The leucocyte-rich plasma was used to set up primary cultures in $5 \mathrm{~mL}$ Medium 199 (Sigma, St. Louis, MO, USA) supplemented with 10\% fetal calf serum (FBS Superior, Biochrom, Berlin, Germany),1\% antibiotic antimycotic solution (Sigma), Lipopolysaccharides (LPS) from E. coli (0.5 mg per 5 $\mathrm{mL}$ medium), Phytohemagglutinin (PHA-P) $(90 \mu \mathrm{g}$ per $5 \mathrm{~mL}$ medium, Remel, Lenexa, KS, USA), kanamycin ( $0.3 \mathrm{mg}$ per $5 \mathrm{~mL}$ medium, Sigma), and $0.175 \mu \mathrm{L} \mathrm{10 \%} \mathrm{mercaptoethanol} \mathrm{(Sigma).}$ After $120 \mathrm{hr}$ of incubation at $20^{\circ} \mathrm{C}, 5 \mathrm{~mL}$ cultures were harvested using standard colchicine (two drops of $0.1 \%$ colchicine per $5 \mathrm{~mL}$ media) and $0.075 \mathrm{M} \mathrm{KCl}$ hypotonic ( $8 \mathrm{~min}$.) treatments followed by fixation in a freshly prepared fixative (methanol: acetic acid 3:1, v/v) three times. Cell suspensions of Anolis carolinensis, Eremias velox, and Gallus gallus were obtained by leucocyte cultivation sensu Sohn and Ryu ('99) and Pokorná et al. (2010) with slight modifications. Chromosome preparations of M. musculus were obtained from bone marrow sensu Ford and Hamerton ('56).

All national guidelines for the care and use of animals were followed. This study was covered by the "Valid Animal Use Protocols" Nr. CZ00221 of the Laboratory of Fish Genetics, IAPG, issued by the Czech Ministry of Agriculture; by the valid Mexican permission SAGARPA/CONAPESCA, DGOPA 09004.04111.3088 and by the Animal facilities accreditation of the Faculty of Science, Charles University in Prague, Czech Republic (24773/200810001).

$\mathrm{CMA}_{3} / \mathrm{DAPI}(\mathrm{CDD})$ Staining. Chromomycin $\mathrm{A}_{3}\left(\mathrm{CMA}_{3}\right.$, DNA dye-specific for GC-rich regions) and DAPI (AT-specific) fluorescent staining was performed as described by Sola et al. ('92) on metaphases of the following species: A. tropicus, L. oculatus, A. calva, A. stellatus, $R$. rutilus, A. anguilla, P. ornatipinnis, $P$. lapradei, P. lessonae, A. carolinensis, E. velox, G. gallus, and M. musculus.

G-and C-Banding. G-banding was performed sensu Seabright ('71, '72) on the same chromosome preparations treated with CDD staining to directly compare banding patterns. During Gbanding, we tested 15, 20, and 30 sec of trypsin treatment. Gbanding was tested on chromosomes of Amia calva, Acipenser baeri, A. gueldenstaedtii, A. ruthenus, and A. stellatus.

C-banding was performed sensu Sumner ('90) with the slight modifications described in Pokorná et al. (2014) to determine whether heterochromatin accumulations resemble the Gbanding pattern as described previously (Schmid and Guttenbach, '88; Graphodatsky, '89; Holmquist and Ashley, 2006). Chromosomes were counterstained by DAPI to enhance contrast. Microphotographs were taken in the fluorescent regime and inverted. Chromosomes were classified sensu Levan et al. ('64). Further details on the molecular cytogenetic and image analyses are listed in the Supporting Information.

\section{Bioinformatic Analysis}

We calculated GC profiles for each linkage group (LG, are expected to correspond to chromosomes, Braasch et al., 2016) in L. oculatus and compared them with GC profiles of Danio rerio, Tetraodon nigroviridis, and Gasterosteus aculeatus. For comparison with representative mammalian genomes, we calculated GC profiles of the house mouse and human. The profiles were calculated with a sliding window of $10 \mathrm{kbp}$, striding the chromosome in $10 \mathrm{kbp}$ steps. Profile plots were made after combining the original data to $100 \mathrm{kbp}$ windows. Genome assemblies of analyzed species were downloaded from www.ensembl.org (version published in Flicek et al., 2014). 
In addition, for the spotted gar genome, an analysis of GC content in genes versus intergenic regions was conducted. This analysis was performed twice, once with all sequence data and once with transposons and low-complexity regions excluded from the intergenic regions. To identify the genomic regions, transposons, and low-complexity regions, we relied on annotations provided by ENSEMBL.

Genome assemblies were preprocessed using Python, in that A/C/G/T bases were counted for each genome. These data sets were then subjected to a GC content analysis performed for each LG separately using $R$ v. 3.0, packages ggplot, dplyr, tidyr ( $R$ Development Core Team, 2012). All custom-generated Python and $\mathrm{R}$ scripts are available on the codes repository GitHub (https://github.com/libor-m/vertebrate-GC).

\section{RESULTS}

Alternation of AT- and GC-Rich Regions on Gar Chromosomes

In $A$. tropicus, the diploid chromosome number was $2 n=56$, the chromosomal arms number $\mathrm{NF}=90$ in both females and males (Fig. 2A). The karyotype was composed of 18 pairs of macrochromosomes and 10 pairs of very small chromosomes (Figs. 2A, C, E, and G; marked as st/a*). The chromosomes consisted of five pairs of meta- $(\mathrm{m}), 11$ pairs of submeta- $(\mathrm{sm})$, and 12 pairs of subtelo- (st) to acrocentric (a) chromosomes (Figs. 2A, C, E, and $\mathrm{G})$. In metaphases with less condensed chromosomes after CDD staining, two pairs of biarmed and eight pairs of uniarmed very small chromosomes could be distinguished among the st/a category. There were no detectable differences between males and females.

In L. oculatus, $2 n=58$ in unsexed animals, NF $=92$, the karyotype composed of 19 pairs of macrochromosomes and 10 pairs of very small chromosomes (Figs. 2B, D, F, and H; marked as st/a*). Chromosomes consisted of 4 pairs of metacentrics, 10 pairs of submetacentrics, and 14 pairs of subtelo- to acrocentric chromosomes (Figs. 2B, D, F, and H). In less condensed metaphases after CDD staining, two pairs of bi- and eight pairs of uniarmed very small chromosomes could be distinguished among the st/a category.

The base-specific CDD (DAPI/CMA $)_{3}$ staining of chromosomes of A. tropicus and L. oculatus demonstrated a clear $\mathrm{CMA}_{3}{ }^{+}$ banding pattern (GC-rich regions) along with a $\mathrm{DAPI}^{+}$pattern (AT-rich regions). This alternation of AT/GC bands enabled the unambiguously distinguishing between homologous and homeologous chromosomes, excluding the very small (the ten smallest) chromosomes (Figs. 2A and B). Giemsa-stained karyotypes of A. tropicus and L. oculatus are shown in Figures $2 \mathrm{G}$ and $\mathrm{H}$. This banding pattern enabled us to generate ideograms for both species (Fig. S1a and b in the Supporting Information). Fluorescence in situ hybridization (FISH) with rDNA yielded two ( $A$. tropicus) or three (L. oculatus) signals for $28 \mathrm{~S}$ rDNA and two signals for $5 \mathrm{~S}$ rDNA were detected in both species (Fig. S2 in the
Supporting Information). Contrary to gars, all other ray-finned fishes included in this analysis showed a "teleost pattern" with homogeneously stained chromosomes, that is a balanced proportion of AT/GC and GC-rich rDNA sites (Figs. 3C-G). The same pattern was observed in amphibians represented by $P$. lessonae (Fig. 3H). In reptiles, we identified a homogeneous pattern in Anolis carolinensis (Fig. 3J) and indications of compositional heterogeneity in Eremias velox (Fig. 3I). In the chicken, CDD staining yielded mostly homogeneously stained macrochromosomes (some with $\mathrm{GC}^{+}$regions terminally) and GC-rich microchromosomes (Fig. 3K). In the mouse, the pattern of alternating $\mathrm{AT}^{+} / \mathrm{GC}^{+}$bands occurred on all chromosomes (Fig. 3L; details are given in the Supporting Information).

\section{Heterochromatic C-Bands Do Not Mimic G-Bands}

G-banding in gars produced a pattern enabling the karyotyping of chromosomes (Figs. 2C and D). The G-banding pattern corresponds to the CDD pattern: The positive G-bands (dark; sensu Sumner, '90) match up with the AT-rich bands, and the negative G-bands (pale; sensu Sumner '90) match up with the GC-rich bands. No clear G-banding pattern was observed in sturgeons and bowfin (not shown). Further details are given in the Supporting Information.

C-banding, which is used to visualize constitutive heterochromatin, produced bright signals in the GC-rich centromeres and weaker signals interstitially in L. oculatus and A. tropicus (Fig. 2E and F).

Based on our results, we can summarize the relationship between the constitutive heterochromatin (the C-bands), G-bands, and AT-/GC-rich regions in gars as follows: (i) The centromeric constitutive heterochromatin overlaps with the GC-rich centromeric regions but does not overlap with G-bands that are mostly absent in centromeres; (ii) the usually terminally located GC-rich regions (Fig. 2; e.g., the 1. and 2. metacentric, the 3. and 4. submetacentric chromosomes) are clearly C-negative as well as G-negative; (iii) the interstitial GC-rich bands appear to be present in the vicinity of the C-bands; however, this finding needs to be verified by specialized analysis; (iv) The G-bands can be found in clearly C-negative, C-positive, and slightly Cpositive regions; (v) The very GC-rich, and also to a large extent heterochromatinized, chromosomal pair is G-positive on the $q$ arm. Details are described in the Supporting Information. This indicates the euchromatic genome occupation of a significant proportion of G-bands in gars similar to the situation in warmblooded vertebrates (sensu Bickmore and Craig, '96). By comparing the results with the CDD patterns, we can rule out that the C-bands mimic the G-banding pattern in gars.

GC Profiles in Genomes of Spotted Gar and Other Relevant Species GC profiles of LGs of the spotted gar (L. oculatus) genome appear to correspond to our cytogenetic results and demonstrate the AT/GC heterogeneity observed along gar chromosomes at 


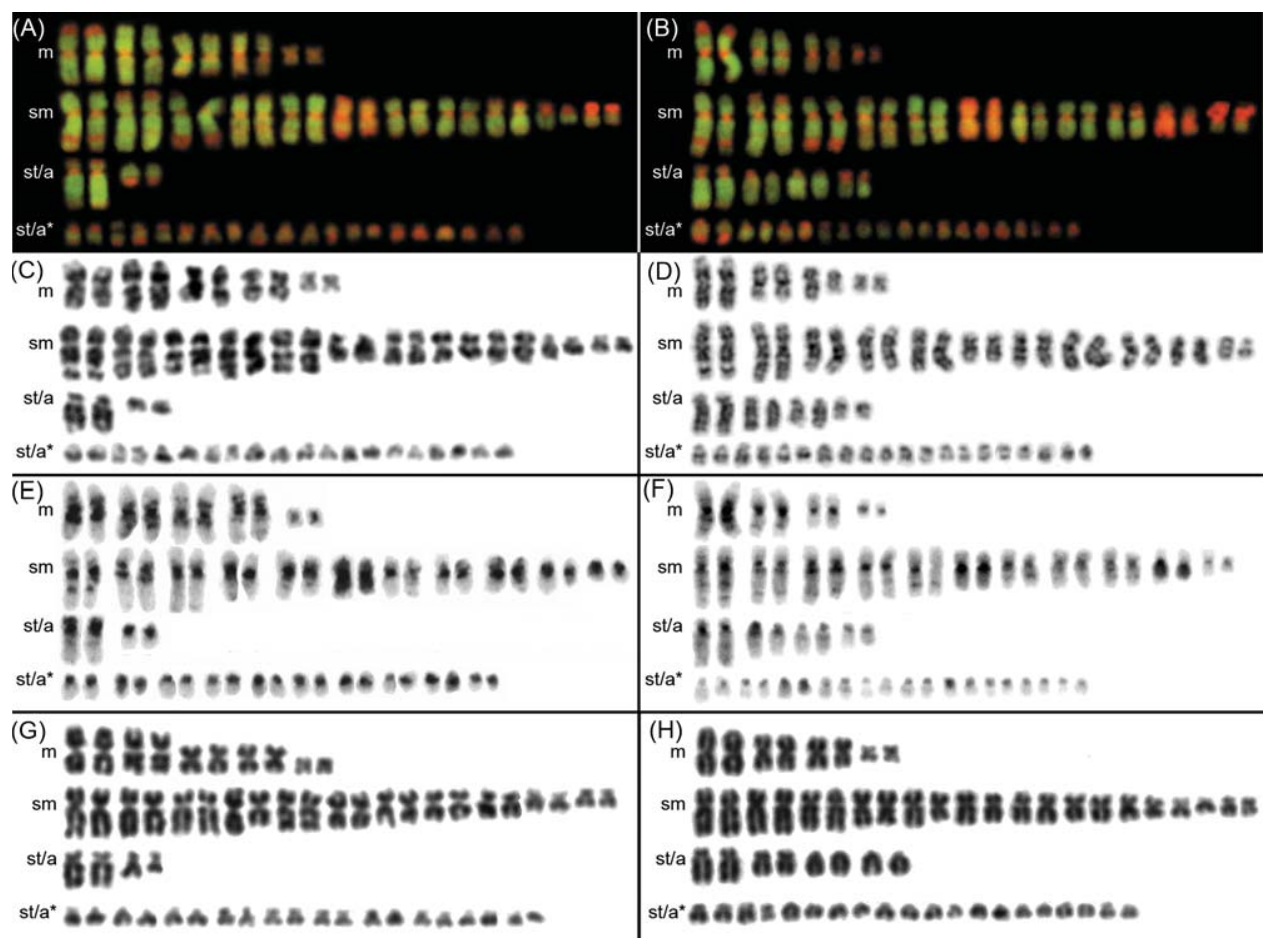

Figure 2. Karyotypes of tropical gar (A) CDD-stained; (C) G-banded; (E) C-banded; (G) Giemsa stained and of spotted gar (B) CDD-stained; (D) G-banded; (F) C-banded; (H) Giemsa stained. The $\mathrm{CMA}_{3}$ signal was inserted from green into red and the DAPI signal from blue into the green channel. The $\mathrm{C}$-banding pattern was counterstained with DAPI and inverted. $\mathrm{m}$, metacentric; sm, submetacentric; st/a, subtelocentricacrocentric chromosomes; st/a*, subtelocentric-acrocentric very small chromosomes. Images were adjusted separately before being incorporated into the plate. [Color figure can be viewed at wileyonlinelibrary.com]

the sequence level (Fig. 4A). The GC profiles also enabled the quantification of the qualitative cytogenetic data showing an alternation of GC-rich and GC-poor regions along chromosomes. Bernardi (2005) defines "heavy" GC-rich DNA domains as having $>45 \%$ GC and "light" domains with 35-45\% GC (Fig. 4A). The GC-rich regions in L. oculatus frequently exceeded 50\% GC over DNA regions of several Mbp, for example, in LG1-2, LG46, LG8-12, and most of the small chromosomes. The GC-rich and AT-rich regions form discernible compositionally homogeneous domains. The AT-rich regions form flattened stretches. The GC-rich regions occur as GC-rich peaks interrupted by sharp decreases in GC content. The GC-rich regions are mostly telomerically but also interstitially situated and correspond to the GCrich bands after CDD staining. However, it was impossible at this stage to identify exactly which linkage group of the $L$. oculatus genome represents which pair of chromosomes, since there were no identification markers present to link both types of data sets. GC profiles of LGs in the pufferfish with a compact teleost genome and a shift toward a higher GC content show a pattern of very densely distributed fluctuations between 40 and 50\% GC with terminal (telomeric) sharp narrow increases up to 55-60\% of GC (Fig. 4B). GC profiles of the stickleback also show a slight shift toward a higher GC content, compositional fluctuations between 40 and 50\% GC, no prominent GC peaks and several regions of profile flattened around 45\% GC (Fig. 4C). GC profiles of the zebrafish (Danio rerio), representing homogenized teleost genomes, are distinctly flattened between 35 and 40\% GC. Only very narrow peaks reaching up to 50\% GC occur in the terminal locations in the majority of LGs (Fig. 4D). In the house mouse and human, the same procedure shows fluctuations in the range of 35-55\% GC, with the exception of the Y chromosome with a narrow compositional range of around 40\% GC (Figs. 4E and F; Figs. S5a and b (in the Supporting Information)). Comparable patterns were obtained with window lengths of 10, 250, 500, and $1000 \mathrm{kbp}$ (not shown).

The analysis of GC content in genes versus intergenic regions of the spotted gar and other tested actinopterygian genomes shows that gene-rich regions are more GC-rich in the spotted gar as well as in pufferfish and stickleback, but not in zebrafish (Figs. S4a-d in the Supporting Information). In the spotted gar, the gene-rich regions contain $25-75 \%$ GC, whereas the intergenic regions 0-55\% GC. Both the genic and intergenic 

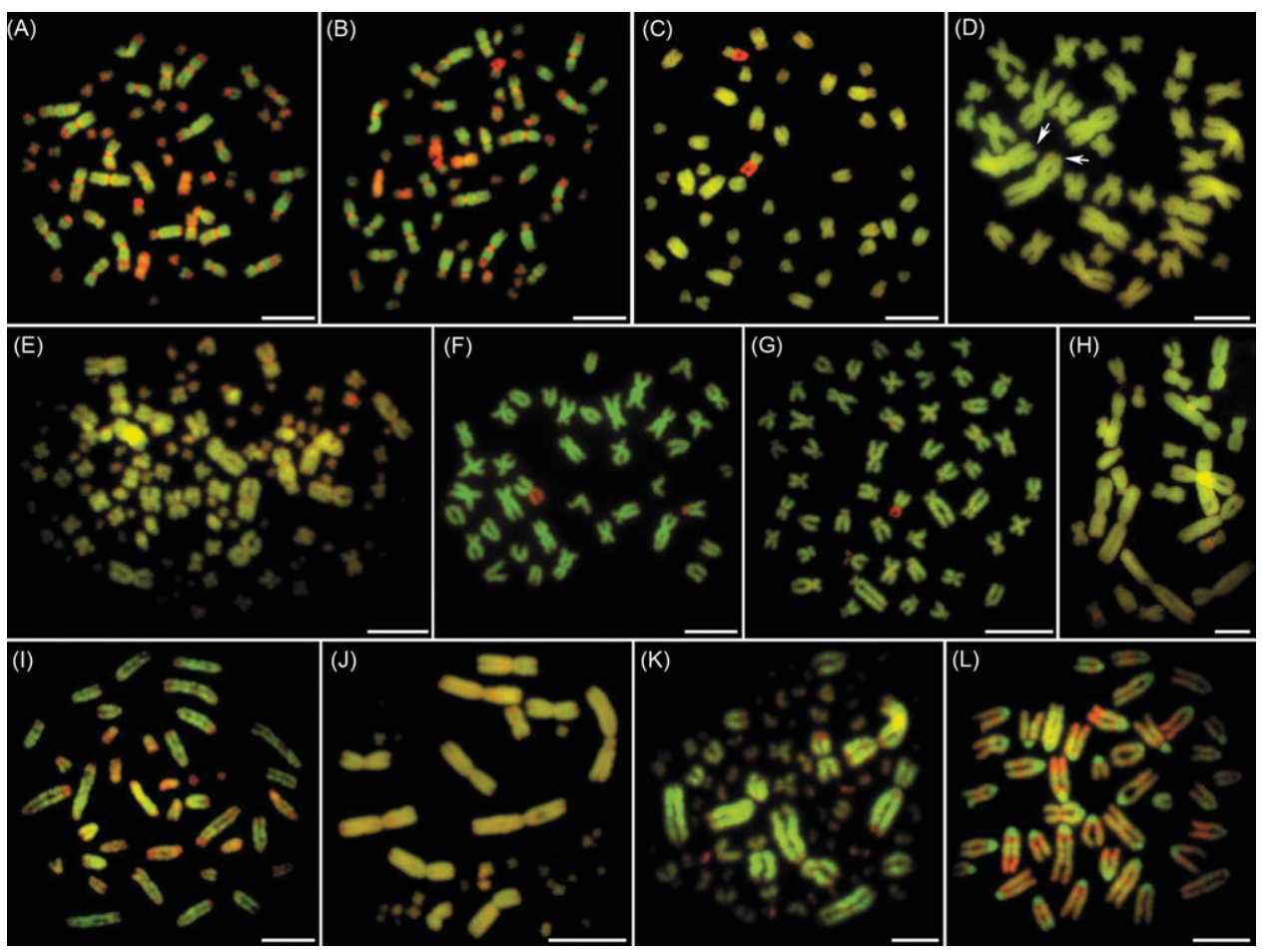

Figure 3. CDD-stained chromosomes of different vertebrate representatives: (A) tropical gar Atractosteus tropicus; (B) spotted gar Lepisosteus oculatus; (C) bowfin Amia calva; (D) ornate bichir Polypterus ornatipinnis; (E) starry sturgeon Acipenser stellatus; (F) European eel Anguilla anguilla; $(\mathrm{G})$ roach Rutilus rutilus; $(\mathrm{H})$ pool frog Pelophylax lessonae; (I) rapid racerunner Eremias velox; $(\mathrm{J})$ green anole Anolis carolinensis; $(\mathrm{K})$ chicken Gallus gallus and $(\mathrm{L})$ house mouse Mus musculus. The $\mathrm{CMA}_{3}$ signal was inserted from green into red and the DAPI signal from blue into the green channel to enhance the contrast between these signals. Images were adjusted separately before being incorporated into the plate. Bars equal $5 \mu \mathrm{m}$. [Color figure can be viewed at wileyonlinelibrary.com]

regions show maximal density at approximately 38\% GC in the spotted gar (Fig. S4a in the Supporting Information). The GC-richness of gene-rich regions is even more pronounced in stickleback and pufferfish (Figs. S4b and c in the Supporting Information). In both of these species, the maximal density in genic regions shows a shift toward higher GC content when compared with the intergenic regions (Figs. S4b and c in the Supporting Information).

\section{DISCUSSION}

Cytogenetically Detectable AT/GC Compartmentalization in Gars CDD staining is a versatile and well-understood tool visualizing the global AT/GC distribution in plant and animal chromosomes (Sumner, '90). This is not the case for G-banding where uncertainties about exact interactions between the dye and DNA persist (Sumner, '90). In gars, we have combined both these stainings and excluded that constitutive heterochromatin mimics G-bands by C-banding in both gar species (Figs. 2E and F).
This was further supported by bioinformatic analyses showing that GC-rich regions are more gene-rich (Fig. S4 in the Supporting Information). So far, eels were the only exception among fishes with repeatedly reported functioning G-bands (Wiberg, '83; Sola et al., '84). However, our CDD staining in the eel yielded the "typical teleost pattern," that is without any GC heterogeneity along chromosomes up to two $\mathrm{GC}^{+}$rDNA sites (Fig. 3F). Equilibrium centrifugation in eels also did not show any pattern similar to the GC-heavy isochores (Bernardi, 2005) characteristic for birds and mammals. A. anguilla exhibits an intermolecular compositional heterogeneity (Medrano et al., '88; Bernardi and Bernardi, '90) similar to what was found in A. rostrata and explained by the presence of GC-rich satellites (Hudson et al., '80). Medrano et al. ('88) admitted that the G-bands they had produced in eels were not of the same quality as the results produced by the same technique in mammals. Moreover, CDD staining in several anguilliform fishes demonstrated that rDNA sites are the only GC-rich regions on otherwise homogeneously stained chromosomes (Salvadori et al., 2009; Coluccia et al., 2010). Therefore, 


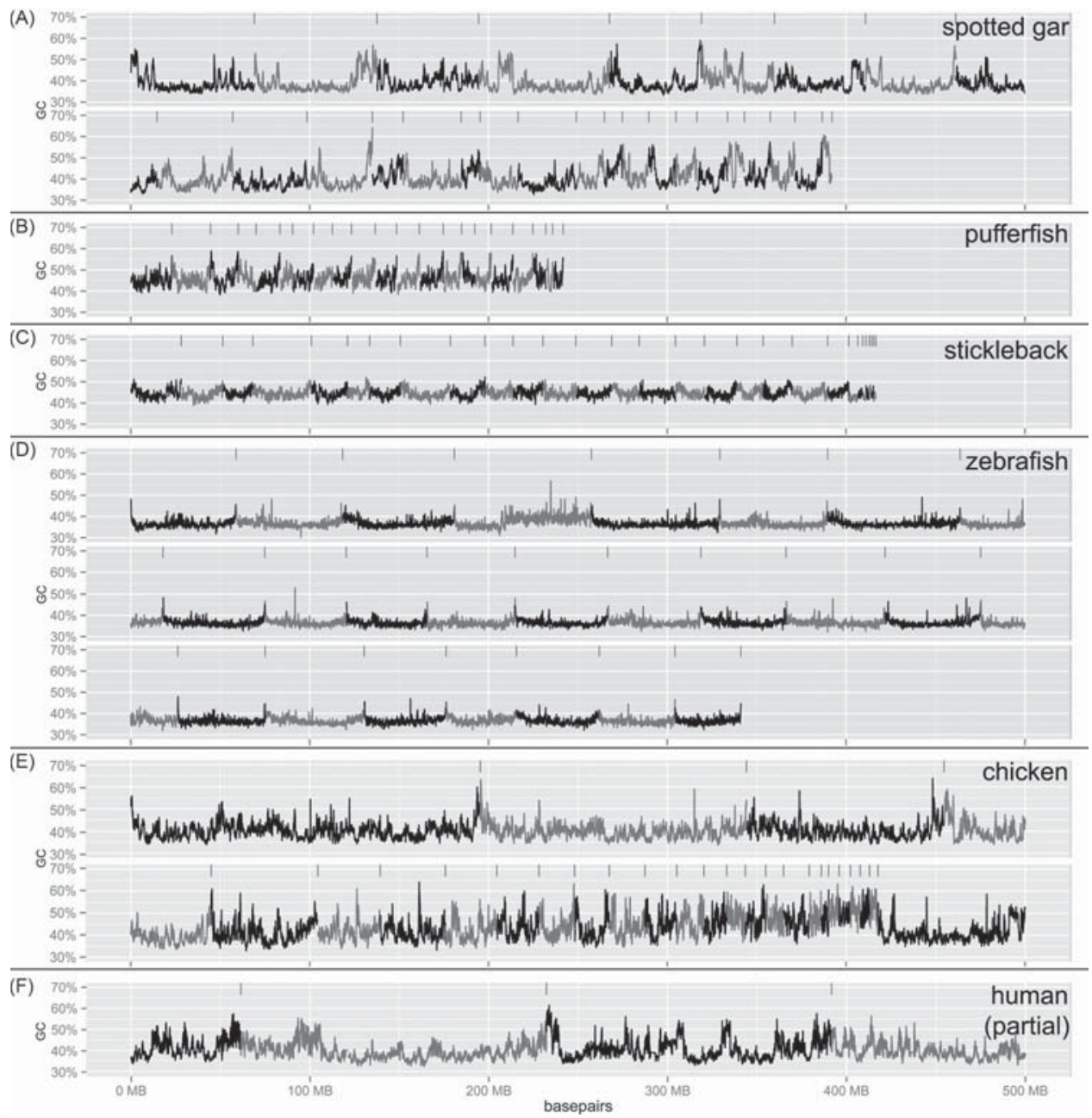

Figure 4. GC profiles along linkage groups (LGs) in (A) spotted gar, (B) pufferfish, (C) stickleback and (D) zebrafish. For comparison, chicken (E) and a partial human (F) GC profile are provided (for full human GC profile, see Fig. S3a in the Supporting Information). GC profiling was performed with uniform scaling, and differing genome sizes are reflected in sizes of profiles. LGs are arranged according to their numbers in the Ensembl database and separated by vertical lines above profiles and by the alternation of gray and black colors ( $x$ axis - genome position, $y$ axis - percentage of GC).

no reliable report has yet been published on the successful application of G-banding in fishes as also noted by Sharma et al. (2002).

For anurans, Bernardi (2005) summarized equilibrium centrifugation results in Xenopus, Leptodactylus, Bufo, and Rana and one representative of the Caudata (Pleurodeles waltlii) and concluded that the genome compositional properties of Amphibians are similar to those described for fishes (Bernardi, 2005). Gazoni et al. (2012) performed CDD staining in eight species of the Leptodactylus frog and showed a pattern similar to the ones we observed in P. lessonae (Fig. S1 in the Supporting Information) and teleosts in this study. Reproducible G-banding was never successful in anurans, whereas replication banding was routinely possible (Schmid and Steinlein 2015).

There is a comparable pattern of compositional heterogeneity in gars and the house mouse with similar counts of discernible AT/GC bands per chromosome differing in AT-rich centromeres in the mouse and GC-rich in gars (Fig. 4L).

The G-bands previously reported in teleosts should be considered "G-like" bands unrelated to the genome GC compartmentalization observed in homeotherm vertebrates. We ascribe these G-like bands to a specific phase of DNA spiralization (less condensed), when some chromosomal regions are more susceptible to Giemsa staining and/or trypsin digestion. 
Nevertheless, the alternation of early and late replicating DNA regions exists also in cold-blooded vertebrates as demonstrated by replication banding in fish and amphibians (Vinas et al., '96; Jankun et al., '98; Boroń, 2003; Schmid and Steinlein, 2015). The structural role of gene-rich and gene-poor regions sensu Cremer and Cremer (2001) appears to be universal across all vertebrates (Federico et al., 2006). Hence, the genome organization in coldblooded vertebrates is not accompanied with such prominent GC segregation as in warm-blooded genomes. Even in species with more GC heterogeneous genomes (pufferfish, stickleback), the base distribution appears more shuffled and without accumulation in any distinctly GC homogeneous (GC-rich or GC-poor) domains. This may be the reason why it is impossible to visualize any bands in fish chromosomes (with the exception of gars). Therefore, it is necessary to distinguish between the ranges of GC values represented in fish genomes globally and between the AT/GC distributions along chromosomes. In anamniotes, another important factor can be the fact that noncoding sequences with various GC content may blur the higher GC content of coding sequences (Fortes et al., 2007). This is particularly apparent in Figs. S4 a-d in the Supporting Information, where stickleback and pufferfish represent genomes with reduce genome size (for comparison of genome sizes Figs. 4A-D) and a clear shift toward GC-richness. This shift is however not accompanied by such pronounced GC heterogeneity as in gars and amniotes.

How Did the GC Heterogeneity in Gar Arise?

DNA nucleotides are not randomly distributed within genomes (Straalen and Roelofs, 2012). Currently, six mutually nonexclusive hypotheses, with different weight, have been proposed to explain the variation in AT/GC organization between cold- and warm-blooded vertebrates: (1) the thermodynamic stability hypothesis, (2) selection for GC content, (3) AT-biased mutations, (4) GC-biased gene conversion (gBGC), (5) DNA methylation (Mugal et al., 2015a), and (6) the footprint of metabolism (Berná et al., 2012). With our cytogenomic data and literature sources, we can assess three of these hypotheses: (1), (4), and (6).

\section{Thermostability Hypothesis}

The "thermostability hypothesis" (Bernardi, 2005 for review) explains the higher GC heterogeneity of warm-blooded genomes as an adaptation to increased body temperature, where higher GC levels contribute to the stabilization of coding DNA fractions, RNAs, and proteins (Costantini et al., 2009). Specifically, the stabilization of GC-rich and gene-rich open chromatin structures with their high transcriptional activity associated with an increased DNA bendability and decreased nucleosome formation potential (Bernardi, 2005; Vinogradov, 2005). It has not been explicitly tested whether gars are cold- or warmblooded (Miller et al., 2006; Nelson, 2006). However, since they are actinopterygians (Cavin, 2010; Near et al., 2012), they are definitely anamniotes which rules out the possibility that GC heterogenization occurred at the anamniotes - amniotes transition. This opposes the "thermostability hypothesis" linking GC heterogenization with the homeothermy of amniotes (Costantini et al., 2009; Uliano et al., 2010; Tarallo et al., 2016). Based on the report of Farrell (2007), we assume that gars are coldblooded. The tropical gar in Mexico is able to tolerate the extremely high temperatures and very low oxygen conditions of tropical swamps (Burleson et al.,'98; Miller et al., 2006; AriasRodriguez own observations). Whereas, gars from the temperate regions of North America, including the spotted gar analyzed in this study, thrive also in low temperatures. Further investigations should be carried out to study their thermoregulation adaptations, as for example, in elasmobranchs and tuna fish (Block and Finnerty, '94).

\section{GC-Biased Gene Conversion}

GC-biased gene conversion (gBGC) belongs to widely evidenced hypotheses and states that regions with higher recombination rates contain higher local GC content (Lartillot 2013; Mugal et al., 2013, 2015b). In gars, the centromeres, the majority of very small chromosomes, and some short chromosomal arms (all anticipated high-recombining regions), together with a number of other regions, are distinctly GC-rich (Fig. 2A and B; Figs. S3a and $\mathrm{b}$ in the Supporting Information). This supports gBGC in terms introduced, for example, by Pessia et al., 2012, who successfully used chromosome size as a proxy for recombination rate. High GC levels also occur in high-recombining $45 \mathrm{~S}$ rDNA regions in gars, which is consistent with the gBGC model demonstrated by Escobar et al. (2011). Consequently, low recombination rates are associated with decreased GC proportions, for example, in the short-tail opossum where the low number of large chromosomes was proven to be linked with the low recombination rate in autosomes and an overall low GC content (Mikkelsen et al., 2007). We show similar situations in the pool frog and the anole, where the large chromosomes were homogeneously CDD-stained (Fig. 3). The same correlation between chromosome length and GC distribution has been reported in birds and mammals (Romiguier et al., 2010; Weber et al., 2014).

\section{Gars' Unique Metabolism}

A growing number of studies, some also in teleosts (Uliano et al., 2010; Chaurasia et al., 2011, 2014; Berná et al., 2012; Tarallo et al., 2016) introduced a novel hypothesis that environment temperature, oxygen content, and lifestyle may be factors correlating with the genome GC composition. Specifically, an increment in metabolic rate, known to be higher in mammals and birds, affects transcription activity and chromatin structure (Berná et al., 2012).

This hypothesis may be of potential importance in the future since in the Florida gar, the following metabolic adaptations were identified: (i) a heightened capacity to synthesize and oxidize glucose; (ii) a heightened capacity for anaerobic 
glycolysis; (iii) a low number of oxidative enzymes in gar tissues, and (iv) the evolution of their modified swim bladder resembling the lungs of tetrapods in form and function (Frick et al., 2007; Echelle and Grande, 2014 and references therein). Gars are bimodal in respiration-they use their gills for water breathing and their lung(s) for air breathing. This gives them a greater respiratory efficiency in exhaustive situations, allowing them to continue normal activity under conditions that would incapacitate virtually every other fish (Echelle and Grande, 2014).

Chaurasia et al. (2014) proposed a correlation between temperature-corrected metabolic rate (MR), entire genomic GC content, and the length and GC content of introns in teleosts. This will also have to be tested in gars. Hence, the overall proportion of introns and their GC content may indirectly influence the GC content of the entire genome (e.g., in compact genomes). In this regard, gars may differ from other tropical fish in their modified metabolism and/or increased MR (Uliano et al., 2010). Therefore, the temperature component might be involved in the compositional genome evolution as suggested by Bernardi (2005). However, the final modulation might instead be influenced by metabolism-driven traits (Uliano et al., 2010; Chaurasia et al., 2011). These correlations and potential causes are yet to be properly analyzed, particularly in gars and bowfin. Differences in physiology between gars and the bowfin indicate specific metabolic traits in gars resembling those of mammals (Frick et al., 2007). This might help to explain why gars possess cytogenetically and bioinformatically detectable GC heterogeneity whereas their putative sister lineage represented by the bowfin exhibit the typical "teleost pattern" on its chromosomes.

\section{Evolutionary Rate in Gars' Evolution}

Gars are considered to have evolved slowly and to show low rates of speciation (Rabosky et al., 2013; Braasch et al., 2016). However, these analyses are based only on the living subset of vertebrate lineages, overlooking the great diversity seen in the fossil record, more so than that found in Polypteriformes, Chondrostea, and Amiidae (Cavin, 2010; López-Arbarello 2012; Sallan, personal communication). The palaeobiology of gars and the bowfin based on solid fossil evidence (Sallan, personal communication) indicates a 50 million year long period of accelerated evolution that occurred in gars but not in amiids with an otherwise very similar evolutionary-ecological history (Grande, 2010). This period of accelerated evolution might have also been accompanied by dynamic changes in gars, which may have left a footprint in their genome in the form of GC compartmentalization. (More details are presented in the Supporting Information). Moreover, based on the fossil evidence, gars and early tetrapods could have faced similar selective pressures and converged on similar solutions (Sallan, personal communication). On the other hand, at this stage, we also have to take into account that gars could have retained the ancestral genome structure of Gnathostomata.
The compositional genome heterogeneity among vertebrates cannot be attributed merely to the transition from anamniotes to amniotes as generally accepted before this study. The genomic DNA base architecture in vertebrates appears to rather result from a interplay of multiple opposing forces, for example, AT-biased mutations and GC-biased gene conversions (e.g., Weller et al., 2014), genome and chromosome sizes (e.g., Romiguier et al., 2010), selection for GC and drift, and by diverse cellular, metabolic (Chaurasia et al., 2014) and environmental (e.g., Uliano et al., 2010; Chaurasia et al., 2011) factors influenced by physical and chemical properties of AT/GC and their role in the regulation of gene expression (Vinogradov, 2005). In gars, such interplay should be explored with regard to their metabolic adaptations (sensu Uliano et al., 2010; Chaurasia et al., 2011, 2014). The situation presented here in gars, in the light of the known genomic traits of their closest extant relatives, implies important consequences for the phylogenomic evolution not only of basal vertebrates but also for amniotes.

\section{ACKNOWLEDGMENTS}

We thank J. Zima for comments about the manuscript, Š. Pelikánová, J. Čechová, and P. Šejnohová of the LFG for assistance in the laboratory, L. Choleva for P. lessonae cell suspensions, M. Altmanová and L. Kratochvíl for help during chromosome preparations of reptiles, R. Černý for providing $P$. lapradei blood samples, T. Kučera for blood samples of $P$. ornatipinnis, K. Trejbalová for the introduction to ultracentrifugation, J. R. Indy for help with A. tropicus, and L. Sallan for the elucidation of paleobiological aspects of the evolution of gars. All colleagues who are acknowledged agreed to the final version of the text.

All custom generated Python and $\mathrm{R}$ scripts produced for this study are available at the codes repository GitHub: https://github.com/libor-m/vertebrate-GC

\section{AUTHOR CONTRIBUTIONS}

R.S. designed the study. R.S. and Z.M. performed cytogenetic analyses and codrafted the manuscript together with P.R., A.M., E.N., and L.B. L.M. performed bioinformatic analyses. L.C. provided the paleobiological context and codrafted the manuscript together with M.J.P. and Z.M. T.K. and L.A.R. participated on cytogenetic analyses. L.A.R., M.J.P., M.F., and M.D. provided chromosome preparations. All coauthors revised the text and agreed to the final version.

\section{LITERATURE CITED}

Amores A, Catchen J, Ferrara A, Fontenot Q, Postlethwait JH. 2011. Genome evolution and meiotic maps by massively parallel DNA sequencing: spotted gar, an outgroup for the teleost genome duplication. Genetics 188:799-808.

Amores A, Force A, Yan YL, Joly L, Amemiya C, Fritz A, Ho RK, Langeland J, Prince $V$, Wang $Y L$, Westerfield $M$, Ekker $M$, Postlethwait 
JH. 1998. Zebrafish hox clusters and vertebrate genome evolution. Science 282:1711-1714.

Berná L, Chaurasia A, Angelini C, Federico C, Saccone S, D'Onofrio G. 2012. The footprint of metabolism in the organization of mammalian genomes. BMC Genomics 13:174.

Bernardi G. 2005. Structural and evolutionary genomics: natural selection in genome evolution. Amsterdam: Elsevier.

Bernardi G, Bernardi G. 1990. Compositional patterns in the nuclear genome of cold-blooded vertebrates. J Mol Evol 31:265-281.

Bickmore WA, Craig J. 1996. Chromosome bands: Patterns in the genome, 1st ed. New York: Springer.

Block BA, Finnerty JR. 1994. Endothermy in fishes: a phylogenetic analysis of constraints, predispositions, and selection pressures. Environ Biol Fishes 40:283-302.

Boron A. 2003. Replication banding patterns in the spined loach, Cobitis taenia L. (Pisces, Cobitidae). Genetica 119:51-55.

Braasch I, Gehrke AR, Smith JJ, Kawasaki K, Manousaki T, Pasquier J, Amores A, Desvignes T, Batzel P, Catchen J, Berlin AM, Campbell MS, Barrell D, Martin KJ, Mulley JF, Ravi V, Lee AP, Nakamura T, Chalopin D, Fan S, Wcisel D, Cañestro C, Sydes J, Beaudry FEG, Sun $Y$, Hertel J, Beam MJ, Fasold M, Ishiyama M, Johnson J, Kehr S, Lara M, Letaw JH, Litman GW, Litman RT, Mikami M, Ota T, Saha $N R$, Williams L, Stadler PF, Wang $H$, Taylor JS, Fontenot , $_{\text {, Ferrara }}$ A, Searle SMJ, Aken B, Yandell M, Schneider I, Yoder JA, Volff J-N, Meyer A, Amemiya CT, Venkatesh B, Holland PWH, Guiguen Y, Bobe J, Shubin NH, Di Palma F, Alföldi J, Lindblad-Toh K, Postlethwait JH. 2016. The spotted gar genome illuminates vertebrate evolution and facilitates human-teleost comparisons. Nat Genet 48:427-437.

Braasch I, Guiguen Y, Loker R, Letaw JH, Ferrara A, Bobe J, Postlethwait JH. 2014. Connectivity of vertebrate genomes: paired-related homeobox (Prrx) genes in spotted gar, basal teleosts, and tetrapods. Comp Biochem Physiol C Toxicol Pharmacol 163:24-36.

Burleson M, Shipman B, Smatresk N. 1998. Ventilation and acid-base recovery following exhausting activity in an air-breathing fish. J Exp Biol 201:1359-1368.

Cavin L. 2010. Diversity of Mesozoic semionotiform fishes and the origin of gars (Lepisosteidae). Naturwissenschaften 97:1035-1040.

Chaurasia A, Tarallo A, Bernà L, Yagi M, Agnisola C, D'Onofrio G. 2014. Length and $G C$ content variability of introns among teleostean genomes in the light of the metabolic rate hypothesis. PloS One 9:e103889.

Chaurasia A, Uliano E, Berná L, Agnisola C, D'Onofrio G. 2011. Does habitat affect the genomic GC content? A lesson from teleostean fish: a mini review. In: Fish ecology. Hauppauge, NY: Nova Science Publishers. p 61-80.

Cohen N, Dagan T, Stone L, Graur D. 2005. GC composition of the human genome: in search of isochores. Mol Biol Evol 22:12601272.

Coluccia E, Deiana AM, Libertini A, Salvadori S. 2010. Cytogenetic characterization of the moray eel Gymnothorax tile and chromosomal banding comparison in Muraenidae (Anguilliformes). Mar Biol Res 6:106-111.
Costantini M, Auletta F, Bernardi G. 2007. Isochore patterns and gene distributions in fish genomes. Genomics 90:364-371.

Costantini M, Cammarano R, Bernardi G. 2009. The evolution of isochore patterns in vertebrate genomes. BMC Genomics 10:146.

Cremer T, Cremer C. 2001. Chromosome territories, nuclear architecture and gene regulation in mammalian cells. Nat Rev Genet 2:292-301.

Crow KD, Smith CD, Cheng J-F, Wagner GP, Amemiya CT. 2012. An independent genome duplication inferred from Hox paralogs in the American paddlefish-a representative basal ray-finned fish and important comparative reference. Genome Biol Evol 4:937953.

Crow KD, Stadler PF, Lynch VJ, Amemiya C, Wagner GP. 2006. The "fish-specific" Hox cluster duplication is coincident with the origin of teleosts. Mol Biol Evol 23:121-136.

Dehal P, Boore JL. 2005. Two rounds of whole genome duplication in the ancestral vertebrate. PLoS Biol 3:e314.

Duret L, Mouchiroud D, Gautier C. 1995. Statistical analysis of vertebrate sequences reveals that long genes are scarce in GC-rich isochores. J Mol Evol 40:308-317.

Echelle AA, Grande L. 2014. Lepisosteidae: Gars. In: Warren ML, Burr BM, editors. Freshwater fishes of North America: Volume 1: Petromyzontidae to Catostomidae. Baltimore: JHU Press. p 243278.

Escobar JS, Glémin S, Galtier N. 2011. GC-biased gene conversion impacts ribosomal DNA evolution in vertebrates, angiosperms, and other eukaryotes. Mol Biol Evol 28:2561-2575.

Farrell AP. 2007. Cardiovascular systems in primitive fishes. In: McKenzie DJ, Farrel AP and Branner CJ, editors. Fish physiology, Vol. 26: Primitive fishes. San Diego, CA: Academic Press. p 53120.

Federico C, Scavo C, Cantarella CD, Motta S, Saccone S, Bernardi G. 2006. Gene-rich and gene-poor chromosomal regions have different locations in the interphase nuclei of cold-blooded vertebrates. Chromosoma 115:123-128.

Flicek P, Amode MR, Barrell D, Beal K, Billis K, Brent $S$, Carvalho-Silva D, Clapham P, Coates G, Fitzgerald S, Gil L, Girón CG, Gordon L, Hourlier T, Hunt $S$, Johnson N, Juettemann T, Kähäri AK, Keenan $S$, Kulesha E, Martin FJ, Maurel T, McLaren WM, Murphy DN, Nag R, Overduin B, Pignatelli M, Pritchard B, Pritchard E, Riat HS, Ruffier M, Sheppard D, Taylor K, Thormann A, Trevanion SJ, Vullo A, Wilder SP, Wilson M, Zadissa $A$, Aken BL, Birney $E$, Cunningham $F$, Harrow J, Herrero J, Hubbard TJP, Kinsella R, Muffato M, Parker A, Spudich G, Yates A, Zerbino DR, Searle SMJ. 2014. Ensembl 2014. Nucleic Acids Res 42:D749-755.

Ford CE, Hamerton JL. 1956. A colchicine, hypotonic citrate, squash sequence for mammalian chromosomes. Stain Technol 31:247251.

Fortes GG, Bouza C, Martínez P, Sánchez L. 2007. Diversity in isochore structure among cold-blooded vertebrates based on GC content of coding and non-coding sequences. Genetica 129:281289. 
Frick NT, Bystriansky JS, Ballantyne JS. 2007. The metabolic organization of a primitive air-breathing fish, the Florida gar (Lepisosteus platyrhincus). J Exp Zool A Ecol Genet Physiol 307:7-17.

Fujita MK, Edwards SV, Ponting CP. 2011. The Anolis lizard genome: an amniote genome without isochores. Genome Biol Evol 3:974-984.

Fujiwara A, Nishida-Umehara C, Sakamoto T, Okamoto N, Nakayama I, Abe S. 2001. Improved fish lymphocyte culture for chromosome preparation. Genetica 111:77-89.

Gazoni T, Gruber SL, Silva APZ, Araújo OGS, Narimatsu H, Strüssmann C, Haddad CFB, Kasahara S. 2012. Cytogenetic analyses of eight species in the genus Leptodactylus Fitzinger, 1843 (Amphibia, Anura, Leptodactylidae), including a new diploid number and a karyotype with multiple translocations. BMC Genet 13:109.

Grande L. 2010. An empirical synthetic pattern study of gars (Lepisosteiformes) and closely related species, based mostly on skeletal anatomy the resurrection of Holostei. Lawrence, KS: American Society of Ichthyologists and Herpetologists.

Graphodatsky AS. 1989. Conserved and variable elements of mammalian chromosomes. In: Halnan CRE, editor. Cytogenetics of animals. Oxon, UK: CAB International Press. p 95-124.

Holmquist GP. 1989. Evolution of chromosome bands: molecular ecology of noncoding DNA. J Mol Evol 28:469-486.

Holmquist GP, Ashley T. 2006. Chromosome organization and chromatin modification: influence on genome function and evolution. Cytogenet Genome Res 114:96-125.

Hudson AP, Cuny G, Cortadas J, Haschemeyer AE, Bernardi G. 1980. An analysis of fish genomes by density gradient centrifugation. Eur J Biochem 112:203-210.

Inoue JG, Miya M, Tsukamoto K, Nishida M. 2003. Basal actinopterygian relationships: a mitogenomic perspective on the phylogeny of the "ancient fish." Mol Phylogenet Evol 26:110-120.

Jankun M, Ocalewicz K, Woznicki P. 1998. Replication, C- and fluorescent chromosome banding patterns in european whitefish, Coregonus lavavetus L. Hereditas 128:195-199.

Lartillot N. 2013. Phylogenetic patterns of GC-biased gene conversion in placental mammals, and the evolutionary dynamics of recombination landscapes. Mol Biol Evol 30:489-502.

Levan A, Fredga K, Sandberg AA. 1964. Nomenclature for centromeric position on chromosomes. Hereditas 52:201-220.

López-Arbarello A. 2012. Phylogenetic interrelationships of ginglymodian fishes (Actinopterygii: Neopterygii). PLoS One 7:e39370.

Ludwig A, Belfiore NM, Pitra C, Svirsky V, Jenneckens I. 2001. Genome duplication events and functional reduction of ploidy levels in sturgeon (Acipenser, Huso and Scaphirhynchus). Genetics 158:1203-1215.

Luo C. 1998. Multiple chromosomal banding in grass carp, Ctenopharyngodon idellus. Heredity 81:481-485.

Medrano L, Bernardi G, Couturier J, Dutrillaux B, Bernardi G. 1988. Chromosome banding and genome compartmentalization in fishes. Chromosoma 96:178-183.

Meyer A, Málaga-Trillo E. 1999. Vertebrate genomics: More fishy tales about Hox genes. Curr Biol 9:R210-213.
Mikkelsen TS, Wakefield MJ, Aken B, Amemiya CT, Chang JL, Duke S, Garber M, Gentles AJ, Goodstadt L, Heger A, Jurka J, Kamal M, Mauceli E, Searle SMJ, Sharpe T, Baker ML, Batzer MA, Benos PV, Belov K, Clamp M, Cook A, Cuff J, Das R, Davidow L, Deakin JE, Fazzari MJ, Glass JL, Grabherr M, Greally JM, Gu W, Hore TA, Huttley GA, Kleber M, Jirtle RL, Koina E, Lee JT, Mahony S, Marra MA, Miller RD, Nicholls RD, Oda M, Papenfuss AT, Parra ZE, Pollock DD, Ray DA, Schein JE, Speed TP, Thompson K, VandeBerg JL, Wade CM, Walker JA, Waters PD, Webber C, Weidman JR, Xie X, Zody MC, Broad Institute Genome Sequencing Platform, Broad Institute Whole Genome Assembly Team, Graves JAM, Ponting CP, Breen M, Samollow PB, Lander ES, Lindblad-Toh K. 2007. Genome of the marsupial Monodelphis domestica reveals innovation in non-coding sequences. Nature 447:167-177.

Miller RR, Minckley WL, Norris SM. 2006. Freshwater fishes of México. Chicago, IL: University of Chicago Press.

Mugal CF, Arndt PF, Ellegren H. 2013. Twisted signatures of GC-biased gene conversion embedded in an evolutionary stable karyotype. Mol Biol Evol 30:1700-1712.

Mugal CF, Arndt PF, Holm L, Ellegren H. 2015a. Evolutionary consequences of DNA methylation on the $\mathrm{GC}$ content in vertebrate genomes. G3 (Bethesda) 5:441-447.

Mugal CF, Weber CC, Ellegren H. 2015b. GC-biased gene conversion links the recombination landscape and demography to genomic base composition. Bioessays 37:1317-1326

Near TJ, Eytan RI, Dornburg A, Kuhn KL, Moore JA, Davis MP, Wainwright PC, Friedman M, Smith WL. 2012. Resolution of ray-finned fish phylogeny and timing of diversification. Proc Natl Acad Sci U S A 109:13698-13703.

Nelson JS. 2006. Fishes of the world, 4th ed. Hoboken, NJ: Wiley.

Neusser M. 2004. Karyotypevolution, Genomorganisation und ZelIkernarchitektur der Neuweltaffen. Dissertation, LMU München: Fakultät für Biologie.

Pessia E, Popa A, Mousset S, Rezvoy C, Duret L, Marais GA. 2012. Evidence for widespread GC-biased gene conversion in eukaryotes. Genome Biol Evol 4:675-682

Pokorná M, Rábová M, Ráb P, Ferguson-Smith MA, Rens W, Kratochvíl L. 2010. Differentiation of sex chromosomes and karyotypic evolution in the eye-lid geckos (Squamata: Gekkota: Eublepharidae), a group with different modes of sex determination. Chromosome Res 18:809-820.

Pokorná M, Rens W, Rovatsos M, Kratochvil L. 2014. A ZZ/ZW sex chromosome system in the thick-tailed Gecko (Underwoodisaurus milii; Squamata: Gekkota: Carphodactylidae), a member of the ancient gecko lineage. Cytogenet Genome Res 142:190196.

Ráb P, Rábová M, Reed KM, Phillips RB. 1999. Chromosomal characteristics of ribosomal DNA in the primitive semionotiform fish, longnose gar Lepisosteus osseus. Chromosome Res Int J Mol Supramol Evol Asp Chromosome Biol 7:475-480.

Rabosky DL, Santini F, Eastman J, Smith SA, Sidlauskas B, Chang J, Alfaro ME. 2013. Rates of speciation and morphological evolution 
are correlated across the largest vertebrate radiation. Nat Commun 4:1958.

R Development Core Team. 2012. R: a language and environment for statistical computing. Vienna, Austria. Available at http://www.Rproject.org/

Romanenko SA, Biltueva LS, Serdyukova NA, Kulemzina Al, Beklemisheva VR, Gladkikh OL, Lemskaya NA, Interesova EA, Korentovich MA, Vorobieva NV, Graphodatsky AS, Trifonov VA. 2015. Segmental paleotetraploidy revealed in sterlet (Acipenser ruthenus) genome by chromosome painting. Mol Cytogenet 8:90.

Romiguier J, Ranwez V, Douzery EJP, Galtier N. 2010. Contrasting GC-content dynamics across 33 mammalian genomes: relationship with life-history traits and chromosome sizes. Genome Res 20:1001-1009.

Sallan LC. 2014. Major issues in the origins of ray-finned fish (Actinopterygii) biodiversity. Biol Rev 89:950-971.

Salvadori S, Coluccia E, Cannas R, Cau A, Deiana AM. 2009. A ZZZW sex chromosome system in the finless eel Dalophis imberbis (Anguilliformes, Ophichtidae). Genetica 135:283-288.

Schmid M, Guttenbach M. 1988. Evolutionary diversity of reverse (R) fluorescent chromosome bands in vertebrates. Chromosoma 97:101-114.

Schmid M, Steinlein C. 2015. Chromosome banding in Amphibia. XXXII. The genus Xenopus (Anura, Pipidae). Cytogenet Genome Res 145:201-217.

Seabright M. 1971. A rapid banding technique for human chromosomes. Lancet 2:971-972.

Seabright M. 1972. The use of proteolytic enzymes for the mapping of structural rearrangements in the chromosomes of man. Chromosoma 36:204-210.

Sharma OP, Tripathi NK, Sharma KK. 2002. A review of chromosome banding in fishes. In: Sobit RC, Obe G, Athwal RS, editors. Some aspects of chromosome structure and functions. Dordrecht, the Netherlands: Springer. p 109-122.

Sohn SH, Ryu EK. 1999. Chromosome preparation from chick leucocyte culture using ficoll treatment. J AgricTechnol Res 12:131-140.

Sola L, Camerini B, Cataudella S. 1984. Cytogenetics of Atlantic eels: C- and G-banding, nucleolus organizer regions, and DNA content. Cytogenet Genome Res 38:206-210.

Sola L, Rossi AR, laselli V, Rasch EM, Monaco PJ. 1992. Cytogenetics of bisexual/unisexual species of Poecilia. II. Analysis of heterochromatin and nucleolar organizer regions in Poecilia mexicana mexicana by $\mathrm{C}$-banding and DAPI, quinacrine, chromomycin $\mathrm{A} 3$, and silver staining. Cytogenet Cell Genet 60:229-235.

Straalen NM van, Roelofs D. 2012. An introduction to ecological genomics. Oxford, UK: Oxford University Press.
Sumner AT. 1990. Chromosome banding. London: Unwin Hyman.

Tarallo A, Angelini C, Sanges R, Yagi M, Agnisola C, D'Onofrio G. 2016. On the genome base composition of teleosts: the effect of environment and lifestyle. BMC Genomics. 17:173.

Taylor JS, Braasch I, Frickey T, Meyer A, Van de Peer Y. 2003. Genome duplication, a trait shared by 22000 species of ray-finned fish. Genome Res 13:382-390.

Thiery J, Macaya G, Bernardi G. 1976. Analysis of eukaryotic genomes by density gradient centrifugation. J Mol Biol 108:219-235.

Ueda T, Naoi H. 2011. BrdU-4Na-EDTA-Giemsa band karyotypes of 3 small freshwater fish, Danio rerio, Oryzias latipes, and Rhodeus ocellatus. Genome 3:531-535.

Uliano E, Chaurasia A, Bernà L, Agnisola C, D'Onofrio G. 2010. Metabolic rate and genomic GC. What we can learn from teleost fish. Mar Genomics 3:29-34.

Vandepoele K, De Vos W, Taylor JS, Meyer A, Van de Peer Y. 2004. Major events in the genome evolution of vertebrates: paranome age and size differ considerably between ray-finned fishes and land vertebrates. Proc Natl Acad Sci U S A 101:1638-1643.

Varriale A. 2014. DNA methylation, epigenetics, and evolution in vertebrates: facts and challenges. Int J Evol Biol 2014:e475981.

Vinas A, Gómez C, Martínez P, Sánchez L. 1996. Replication banding in the chromosomes of the European eel (Anguilla anguilla). Genetica 98:107-110.

Vinogradov AE. 2005. Noncoding DNA, isochores and gene expression: nucleosome formation potential. Nucleic Acids Res 33:559563.

Weber CC, Boussau B, Romiguier J, Jarvis ED, Ellegren H. 2014. Evidence for GG-biased gene conversion as a driver of betweenlineage differences in avian base composition. BMC Genome Biol 15:549.

Weller AM, Rödelsperger C, Eberhardt G, Molnar RI, Sommer RJ. 2014. Opposing forces of A/T-biased mutations and G/C-biased gene conversions shape the genome of the nematode Pristionchus pacificus. Genetics 196:1145-1152.

Wiberg UH. 1983. Sex determination in the European eel (Anguilla anguilla, L.). A hypothesis based on cytogenetic results, correlated with the findings of skewed sex ratios in eel culture ponds. Cytogenet Cell Genet 36:589-598.

Wright JJ, David SR, Near TJ. 2012. Gene trees, species trees, and morphology converge on a similar phylogeny of living gars (Actinopterygii: Holostei: Lepisosteidae), an ancient clade of rayfinned fishes. Mol Phylogenet Evol 63:848-856.

Zhuang ZM, Wu D, Zhang SC, Pang QX, Wang CL, Wan RJ. 2006. Gbanding patterns of the chromosomes of tonguefish Cynoglossus semilaevis Günther, 1873. J Appl Ichthyol 22:437-440. 